\title{
Ternary quarter wavelength coatings for gravitational wave detector mirrors: Design optimization via exhaustive search
}

\author{
V. Pierro ${ }^{1,1,2, *}$ V. Fiumara, ${ }^{3,2}$ F. Chiadini $\odot,{ }^{4,2}$ V. Granata, ${ }^{5,2}$ O. Durante, ${ }^{6,2}$ J. Neilson, ${ }^{1,2}$ C. Di Giorgio, ${ }^{6,2}$ \\ R. Fittipaldi, ${ }^{5,2}$ G. Carapella, ${ }^{6,2}$ F. Bobba, ${ }^{6,2}$ M. Principe ${ }^{\circ},{ }^{1,2,7}$ and I. M. Pinto $\mathbb{\circledR}^{8,2,7}$ \\ ${ }^{1}$ Dipartimento di Ingegneria, DING, Universitá del Sannio, I-82100 Benevento, Italy \\ ${ }^{2}$ INFN, Sezione di Napoli Gruppo Collegato di Salerno, I-80126 Napoli, Italy \\ ${ }^{3}$ Scuola di Ingegneria, Universitá della Basilicata, I-85100 Potenza, Italy \\ ${ }^{4}$ Dipartimento di Ingegneria Industriale, DIIN, Universitá di Salerno, I-84084 Fisciano, Salerno, Italy \\ ${ }^{5}$ CNR-SPIN, c/o Universitá di Salerno, I-84084 Fisciano, Salerno, Italy \\ ${ }^{6}$ Dipartimento di Fisica “Eduardo R. Caianiello," Universitá di Salerno, I-84084 Fisciano, Salerno, Italy \\ ${ }^{7}$ Museo Storico della Fisica e Centro Studi e Ricerche "Enrico Fermi," I-00184 Roma, Italy \\ ${ }^{8}$ Dipartimento di Ingegneria Elettrica e Tecnologie dell'Informazione, DIETI, Universitá di Napoli Federico II, I-80125 Napoli, Italy
}

(Received 11 February 2021; accepted 5 April 2021; published 4 June 2021; corrected 7 July 2021)

\begin{abstract}
Multimaterial optical coatings are a promising viable option to meet the challenging requirements (in terms of transmittance, absorbance, and thermal noise) of next-generation gravitational wave detector mirrors. In this paper we focus on ternary coatings consisting of quarter-wavelength-thick layers, where a third material $\left(H^{\prime}\right)$ is added to the two presently in use, namely, silica $(L)$ and titania-doped tantala $(H)$, featuring higher dielectric contrast (against silica) and lower thermal noise (compared with titania-doped tantala), but higher optical losses. We seek the optimal material sequences, featuring minimal thermal (Brownian) noise under prescribed transmittance and absorbance constraints, by exhaustive simulation over all possible configurations, for different values of the optical density and extinction coefficient of the third material, including the case of amorphous silicon and silicon nitride operating at ambient and cryogenic temperatures. In all cases studied, the optimal designs consist of a stack of $\left(H^{\prime} \mid L\right)$ doublets topped by a stack of $(H \mid L)$ doublets, confirming previous heuristic assumptions, and the achievable coating noise power spectral density reduction factor ranges from $\sim 0.5$ at $290 \mathrm{~K}$ down to $\sim 0.1$ at $20 \mathrm{~K}$. The robustness of the found optimal designs against layer thickness deposition errors and uncertainties and/or fluctuations in the optical losses of the third material is also investigated. Possible margins for further thermal noise reduction by layer thickness optimization, and strategies to implement it, are discussed.
\end{abstract}

DOI: 10.1103/PhysRevResearch.3.023172

\section{INTRODUCTION}

The visibility distance of the currently operating interferometric detectors of gravitational waves is set by thermal (Brownian) noise in the highly reflective dielectric-multilayercoated mirrors of their optical cavities [1].

During the last two decades, the quest for coating materials featuring large dielectric contrast, low optical absorption (and scattering), and low mechanical losses (directly related to thermal noise, in view of the fluctuation-dissipation principle) has been quite intense.

Since the development in 1997 of titania-doped tantala $\left(\mathrm{TiO}_{2}:: \mathrm{Ta}_{2} \mathrm{O}_{5}\right)$ by Laboratoire des Matériaux Avancés (LMA) [2], still used, together with silica $\left(\mathrm{SiO}_{2}\right)$, in the mirrors of the (currently operational) "advanced" laser inter-

\footnotetext{
*pierro@unisannio.it

Published by the American Physical Society under the terms of the Creative Commons Attribution 4.0 International license. Further distribution of this work must maintain attribution to the author(s) and the published article's title, journal citation, and DOI.
}

ferometer gravitational-wave observatory (LIGO) and Virgo detectors $[3,4]$, several potentially interesting coating materials have been investigated (see Ref. [5] for a recent review).

None of them qualifies as a straight substitute for the materials currently in use, but a few of them are better in terms of some properties (e.g., optical density, and/or mechanical losses), while unfortunately worse regarding others.

Amorphous silicon $(a \mathrm{Si})$, in particular, has received much attention, in view of its large refractive index and limited mechanical losses, down to cryogenic temperatures [6]. Its optical losses, on the other hand, are appreciably larger than those of titania-doped tantala and appear to be strongly dependent on the deposition technology [7]. Amorphous silicon has been indicated as a candidate coating material for thirdgeneration $(3 \mathrm{G})$ cryogenic detectors using crystalline silicon for the mirror substrate (presently made of fused silica) and a 1550-nm laser source [8,9].

Silicon nitrides $\left(\mathrm{SiN}_{x}\right)$ have also been proposed as potentially interesting materials, in view of their flexible stoichiometry, which allows one to tune their refractive index in a wide range of values; their ability to accommodate large substrates, via plasma-enhanced chemical vapor deposition (PECVD); and their fairly low mechanical loss angles, down 
to $10^{-4}$ and below, at ambient as well as cryogenic temperatures $[10,11]$. Their optical losses, though, exceed those of currently used materials [12].

In Refs. [13,14] it was first suggested to modify the simplest binary coating design consisting of alternating quarter-wavelength-thick layers of $\mathrm{SiO}_{2}$ and $\mathrm{TiO}_{2}:: \mathrm{Ta}_{2} \mathrm{O}_{5}$, by using a third denser but optically lossier material in the high-index layers closest to the substrate, where the field intensity is usually low enough to make its relatively large optical losses irrelevant. This would allow one to reduce the total number of layers (and hence the coating thermal noise), in view of the larger optical contrast with silica and/or the lower mechanical losses compared with titania-doped tantala. The feasibility of $a \mathrm{Si}$-based ternary coatings has been recently demonstrated [15].

Optical coatings using more than two lossy materials ( $m$-ary coatings, with $m>2$ ) have been studied for a long time (see, e.g., Ref. [16] for a review). However, the design constraints and requirements of the mirrors used by interferometric detectors of gravitational waves are peculiar, especially regarding the key figure of merit represented by thermal (Brownian) noise, making further analysis necessary.

In this paper we implement exhaustive simulations aimed at identifying the structure of the optimal ternary coating design yielding the lowest thermal noise under prescribed (upper) bounds on power transmittance and absorbance, without any a priori assumption, except that all layers are quarter wavelength (QWL).

The paper is organized as follows. In Sec. II we summarize the relevant modeling assumptions used; in Sec. III we introduce the exhaustive procedure used to find the optimum material sequences; in Sec. IV we present and discuss the simulations done referring to two putative third materials; and in Sec. V we present results obtained for realistic materials, namely, amorphous silicon and silicon nitrides, at both ambient and cryogenic temperatures. The structure of the coating design families that comply with the prescribed transmittance and absorbance constraints, the properties of the minimum thermal noise (optimal) ones, including robustness against layer thickness errors, and uncertainties or fluctuations in the third material extinction coefficient are illustrated. Possible margins for further thermal noise reduction by layer thickness optimization, and strategies to implement it, are also discussed. Conclusions follow in Sec. VI.

\section{COATING MODEL}

In this section we summarize the modeling assumptions used throughout this paper, based on the transmission matrix formalism (see, e.g., Refs. [17,18]) and the simplest thermal noise model introduced in Ref. [19].

\section{A. Optical modeling}

Here, an $\exp \left(\imath 2 \pi f_{0} t\right)$ time dependence is understood, $f_{0}$ being the laser light frequency and $\iota$ being the imaginary unit. The optical properties of a multilayer coating can be deduced from its characteristic matrix

$$
\mathbf{T}=\mathbf{T}_{1} \cdot \mathbf{T}_{2} \cdots \mathbf{T}_{N_{T}}
$$

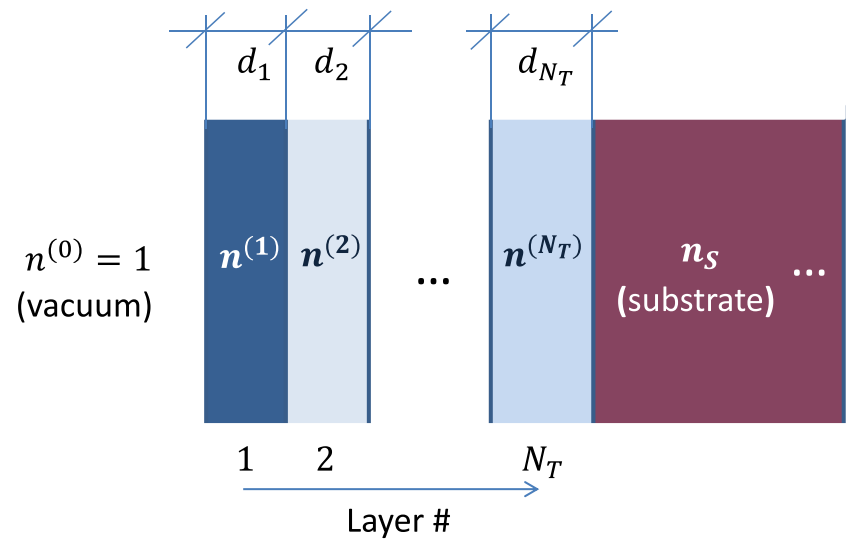

FIG. 1. Sketch of a multilayer coating.

where $N_{T}$ is the total number of layers (numbered from the vacuum to the substrate as in Fig. 1) and $\mathbf{T}_{m}$ is the transmission matrix of the $m$ th layer. Assuming normal incidence [17],

$$
\mathbf{T}_{m}=\left[\begin{array}{cc}
\cos \left(\psi_{m}\right) & \left(l / n^{(m)}\right) \sin \left(\psi_{m}\right) \\
\iota n^{(m)} \sin \left(\psi_{m}\right) & \cos \left(\psi_{m}\right)
\end{array}\right],
$$

where

$$
\psi_{m}=\frac{2 \pi}{\lambda_{0}} n^{(m)} d_{m},
$$

$\lambda_{0}$ and $d_{m}$ being the light free-space wavelength and the $m$ thlayer thickness, respectively, and

$$
n^{(m)}=n_{r}^{(m)}-\imath \kappa^{(m)},
$$

$n_{r}^{(m)}$ being the real refractive index and $\kappa^{(m)}$ being the extinction coefficient of the $m$ th-layer material.

The coating is placed between two homogeneous nondissipative dielectric half-spaces with refractive indices $n^{(0)}$ and $n_{S}$, respectively (see Fig. 1 ). The bottom half-space is the substrate; the top is assumed to be the vacuum, with $n^{(0)}=1$.

The effective complex refractive index of the whole substrate-terminated coating is

$$
n_{C}=\frac{T_{21}+n_{S} T_{22}}{T_{11}+n_{S} T_{12}},
$$

which can be used to compute the (monochromatic plane wave, normal incidence) coating reflection coefficient $\Gamma_{C}$

$$
\Gamma_{C}=\frac{1-n_{C}}{1+n_{C}}
$$

and the power transmittance

$$
\tau_{C}=\frac{\mathcal{P}_{\text {in }}}{\mathcal{P}^{+}}=1-\left|\Gamma_{c}\right|^{2},
$$

where $\mathcal{P}_{\text {in }}$ is the power density flowing into the coating through the vacuum/coating interface and $\mathcal{P}^{+}$is the power density of the incident wave,

$$
\mathcal{P}^{+}=\frac{1}{2 Z_{0}}\left|E_{\mathrm{inc}}\right|^{2},
$$

where $E_{\text {inc }}$ is the (transverse) incident electric field at the vacuum/coating interface and $Z_{0}=\sqrt{\mu_{0} / \epsilon_{0}}$ is the characteristic impedance of the vacuum. 
The average power density dissipated in the coating is the difference between $\mathcal{P}_{\text {in }}$ and the power density flowing into the substrate $\mathcal{P}_{\text {out }}$. This latter can be computed as

$$
\mathcal{P}_{\text {out }}=\frac{1}{2} \operatorname{Re}\left(E^{(S)} H^{(S) *}\right),
$$

where $\operatorname{Re}(\cdot)$ gives the real part of its argument and $E^{(S)}$ and $H^{(S)}$ are the (transverse) electric and magnetic fields at the coating/substrate interface, which are readily obtained from the fields $E^{(0)}=E_{\text {inc }}\left(1+\Gamma_{c}\right)$ and $Z_{0} H^{(0)}=E_{\text {inc }}\left(1-\Gamma_{c}\right)$ at the vacuum/coating interface using the formula

$$
\left[\begin{array}{c}
E^{(S)} \\
Z_{0} H^{(S)}
\end{array}\right]=\mathbf{T}^{-1}\left[\begin{array}{c}
E^{(0)} \\
Z_{0} H^{(0)}
\end{array}\right] .
$$

Accordingly, the coating absorbance is

$$
\alpha_{C}=\frac{\left(\mathcal{P}_{\text {in }}-\mathcal{P}_{\text {out }}\right)}{\mathcal{P}^{+}}=\tau_{C}-\tau_{S},
$$

where

$$
\tau_{S}=\mathcal{P}_{\text {out }} / \mathcal{P}^{+}
$$

is the fraction of the incident power leaking into the substrate. Note that Eq. (11) entails the obvious condition $\tau_{C} \geqslant \tau_{S}$.

\section{B. Thermal noise modeling}

The frequency-dependent power spectral density $S_{\text {coat }}^{(B)}(f)$ of the coating thermal noise can be written

$$
S_{\text {coat }}^{(B)}(f) \propto \frac{T}{w f} \phi_{C},
$$

where $f$ is the frequency, $T$ is the (absolute) temperature, $w$ is the (assumed Gaussian) laser-beam waist, and $\phi_{c}$ is the coating loss angle. Neglecting higher-order terms stemming from subtler effects [20], this latter can be written [19]

$$
\phi_{C}=\sum_{m=1}^{N_{T}} \eta_{m} d_{m}
$$

where

$$
\eta_{m}=\frac{1}{\sqrt{\pi} w} \phi_{m}\left(\frac{Y_{m}}{Y_{S}}+\frac{Y_{S}}{Y_{m}}\right)
$$

is the specific loss angle (loss angle per unit thickness) of the material making the $m$ th layer, $\phi_{m}$ and $Y_{m}$ being its mechanical loss angle and Young's modulus, respectively, and $Y_{S}$ being the Young's modulus of the substrate.

According to Eq. (13), lowering the temperature $T$ would reduce thermal noise [21]. However, in many coating materials, including those currently in use (silica and titania-doped tantala), mechanical losses peak [22,23] in the range of the cryogenic temperatures of interest for next-generation detectors such as the Einstein Telescope (ET) [24] and LIGO-Cosmic Explorer (CE) [25], pioneered by Kamioka Gravitational Wave Detector (KAGRA) [26].

\section{EXHAUSTIVE SCRUTINY OF QWL TERNARY COATINGS}

Exhaustive scrutiny of QWL ternary coatings consists in evaluating the performance (in terms of power transmittance, power absorbance, and coating loss angle) of all (admissible) ternary coatings consisting of QWL layers made of three possible materials, henceforth denoted as $L, H$, and $H^{\prime}$, that comply with given transmittance and absorbance constraints,

$$
\tau_{C} \leqslant \tau_{\text {ref }}, \quad \alpha_{C} \leqslant \alpha_{\text {ref }}
$$

For coatings consisting of QWL layers, the matrices $\mathbf{T}_{m}$, $m=1,2, \ldots, N_{T}$, in (2) take the simple form [27]

$$
\mathbf{T}_{m}=\imath\left[\begin{array}{cc}
\sinh \left(\frac{\pi}{2} \frac{\kappa^{(m)}}{n_{r}^{(m)}}\right) & \frac{1}{n^{(m)}} \cosh \left(\frac{\pi}{2} \frac{\kappa^{(m)}}{n_{r}^{(m)}}\right) \\
n^{(m)} \cosh \left(\frac{\pi}{2} \frac{\kappa^{(m)}}{n_{r}^{(m)}}\right) & \sinh \left(\frac{\pi}{2} \frac{\kappa^{(m)}}{n_{r}^{(m)}}\right)
\end{array}\right] .
$$

For ternary coatings, $n^{(m)}$ can only take values in $\left\{n_{L}, n_{H}, n_{H^{\prime}}\right\}$, and the corresponding single-layer matrices will be denoted as $\mathbf{T}_{L}, \mathbf{T}_{H}$, and $\mathbf{T}_{H^{\prime}}$, respectively.

Among all possible material sequences, those for which $n^{(m)}=n^{(m-1)}$ for some $m$ should be obviously discarded [28]. Accordingly, we are left with a total of $N_{C}=3 \times 2^{N_{T}-1}$ distinct acceptable ternary coatings consisting of $N_{T}$ QWL layers [29].

Knowledge of the matrix (1) yields the coating transmittance and absorbance, as shown in Sect. II.

In order to compute the coating thermal noise, it is expedient to let

$$
\begin{gathered}
\gamma_{H}=\frac{\operatorname{Re}\left[n_{L}\right]}{\operatorname{Re}\left[n_{H}\right]} \frac{\eta_{H}}{\eta_{L}}=\frac{\operatorname{Re}\left[n_{L}\right]}{\operatorname{Re}\left[n_{H}\right]} \frac{\phi_{H}}{\phi_{L}}\left(\frac{Y_{H}}{Y_{s}}+\frac{Y_{s}}{Y_{H}}\right)\left(\frac{Y_{L}}{Y_{s}}+\frac{Y_{s}}{Y_{L}}\right)^{-1}, \\
\gamma_{H^{\prime}}=\frac{\operatorname{Re}\left[n_{L}\right]}{\operatorname{Re}\left[n_{H^{\prime}}\right]} \frac{\eta_{H^{\prime}}}{\eta_{L}} \\
=\frac{\operatorname{Re}\left[n_{L}\right]}{\operatorname{Re}\left[n_{H^{\prime}}\right]} \frac{\phi_{H^{\prime}}}{\phi_{L}}\left(\frac{Y_{H^{\prime}}}{Y_{S}}+\frac{Y_{S}}{Y_{H^{\prime}}}\right)\left(\frac{Y_{L}}{Y_{S}}+\frac{Y_{s}}{Y_{L}}\right)^{-1}
\end{gathered}
$$

where $\phi_{L}, \phi_{H}$, and $\phi_{H^{\prime}}$ are the material mechanical loss angles. Hence, using (14) and (15),

$$
\phi_{C}=\frac{\eta_{L} \lambda_{0}}{4 \operatorname{Re}\left(n_{L}\right)}\left(N_{L}+\gamma_{H} N_{H}+\gamma_{H^{\prime}} N_{H^{\prime}}\right),
$$

$N_{L}, N_{H}$, and $N_{H^{\prime}}$ being the number of layers made of the $L, H$, and $H^{\prime}$ materials, respectively.

The above is a typical constrained optimization problem [30] and has combinatorial complexity. In order to keep the computational burden and computing times within acceptable limits, we use the backtracking strategy [31] to reduce the number of matrix multiplications.

TABLE I. Numerical values of relevant parameters for putative materials A and B. All symbols have the usual meaning.

\begin{tabular}{lcccc}
\hline \hline Property & $\mathrm{SiO}_{2}$ & $\mathrm{TiO}_{2}:: \mathrm{Ta}_{2} \mathrm{O}_{5}$ & Material A & Material B \\
\hline$n_{r}$ & 1.45 & 2.1 & 3.0 & 2.1 \\
$\kappa$ & $10^{-11}$ & $2 \times 10^{-8}$ & $10^{-6}-10^{-4}$ & $10^{-6}-10^{-4}$ \\
$Y(\mathrm{GPa})$ & 72 & 140 & 100 & 100 \\
$\phi$ & $5.0 \times 10^{-5}$ & $3.76 \times 10^{-4}$ & $3.76 \times 10^{-4}$ & $1 \times 10^{-4}$ \\
\hline \hline
\end{tabular}


TABLE II. The set of all admissible ternary QWL coatings subject to (16) using silica for $L$, titania-doped tantala for $H$, and material A for $H^{\prime}$, with $\kappa_{H^{\prime}}=10^{-6}, 10^{-5}, 10^{-4}$. See Sec. III for details. White boxes, $L$; light gray boxes, $H$, dark gray boxes, $H^{\prime}$. The notation $\# H L$ is used to denote the number of $[H \mid L]$ doublets, etc.

\begin{tabular}{|c|c|c|c|}
\hline \multicolumn{4}{|c|}{$\kappa_{H^{\prime}}=10^{-6}$} \\
\hline Structure & $\tau_{C}(\mathrm{ppm})$ & $\alpha_{C}$ (ppm) & $\phi_{c} / \phi_{c}{ }^{(\mathrm{ref})}$ \\
\hline \multicolumn{4}{|l|}{$\left(\# H L, \# H^{\prime} L, \# H^{\prime} H\right)=(0,10,0)$} \\
\hline 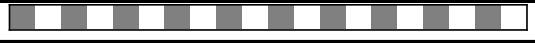 & 2.25 & 0.911 & 0.377 \\
\hline \multicolumn{4}{|l|}{$\left(\# H L, \# H^{\prime} L, \# H^{\prime} H\right)=(1,9,0)$} \\
\hline & 3.19 & 0.463 & 0.395 \\
\hline & 3.53 & 0.806 & 0.395 \\
\hline & 3.61 & 0.886 & 0.395 \\
\hline & 3.63 & 0.905 & 0.395 \\
\hline & 3.63 & 0.910 & 0.395 \\
\hline & 3.64 & 0.911 & 0.395 \\
\hline & 3.64 & 0.911 & 0.395 \\
\hline & 3.64 & 0.911 & 0.395 \\
\hline & 3.64 & 0.911 & 0.395 \\
\hline & 3.64 & 0.911 & 0.395 \\
\hline \multicolumn{4}{|l|}{$\left(\# H L, \# H^{\prime} L, \# H^{\prime} H\right)=(2,8,0)$} \\
\hline & 5.81 & 0.249 & 0.412 \\
\hline & 5.97 & 0.413 & 0.412 \\
\hline \multicolumn{4}{|l|}{$\left(\# H L, \# H^{\prime} L, \# H^{\prime} H\right)=(0,9,1)$} \\
\hline & 3.712 & 0.911 & 0.418 \\
\hline & 3.712 & 0.911 & 0.418 \\
\hline & 3.712 & 0.911 & 0.418 \\
\hline & 3.712 & 0.911 & 0.418 \\
\hline & 3.712 & 0.911 & 0.418 \\
\hline & 3.713 & 0.912 & 0.418 \\
\hline$\square$ & 3.715 & 0.914 & 0.418 \\
\hline$\square$ & 3.725 & 0.924 & 0.418 \\
\hline$\square$ & 3.770 & 0.969 & 0.418 \\
\hline \multicolumn{4}{|c|}{$\kappa_{H^{\prime}}=10^{-5}$} \\
\hline \multicolumn{4}{|l|}{$\left(\# H L, \# H^{\prime} L, \# H^{\prime} H\right)=(4,7,0)$} \\
\hline 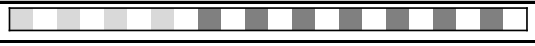 & 5.934 & 0.522 & 0.486 \\
\hline \multicolumn{4}{|c|}{$\kappa_{H^{\prime}}=10^{-4}$} \\
\hline \multicolumn{4}{|l|}{$\left(\# H L, \# H^{\prime} L, \# H^{\prime} H\right)=(7,6,0)$} \\
\hline 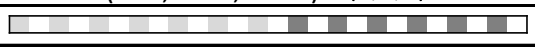 & 3.074 & 0.564 & 0.615 \\
\hline 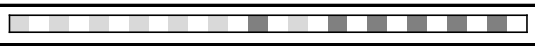 & 3.503 & 0.993 & 0.615 \\
\hline \multicolumn{4}{|l|}{$\left(\# H L, \# H^{\prime} L, \# H^{\prime} H\right)=(8,5,0)$} \\
\hline 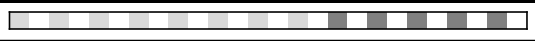 & 5.420 & 0.297 & 0.633 \\
\hline 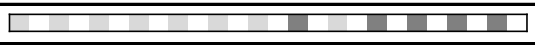 & 5.625 & 0.502 & 0.633 \\
\hline 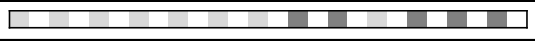 & 5.672 & 0.550 & 0.633 \\
\hline 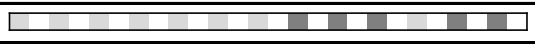 & 5.684 & 0.561 & 0.633 \\
\hline 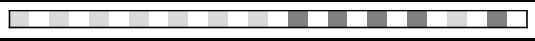 & 5.686 & 0.563 & 0.633 \\
\hline 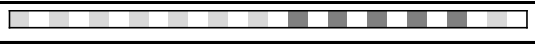 & 5.687 & 0.564 & 0.633 \\
\hline \multicolumn{4}{|l|}{$\left(\# H L, \# H^{\prime} L, \# H^{\prime} H\right)=(7,5,1)$} \\
\hline 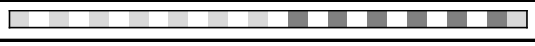 & 5.829 & 0.564 & 0.656 \\
\hline 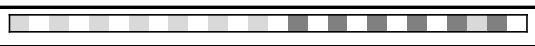 & 5.830 & 0.565 & 0.656 \\
\hline 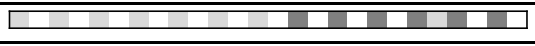 & 5.831 & 0.566 & 0.656 \\
\hline 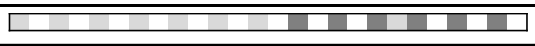 & 5.836 & 0.571 & 0.656 \\
\hline 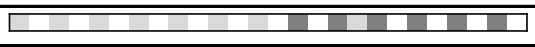 & 5.856 & 0.595 & 0.656 \\
\hline 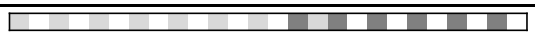 & 5.960 & 0.695 & 0.656 \\
\hline
\end{tabular}

\section{NUMERICAL EXPERIMENTS}

In this section we apply the above-mentioned exhaustive scrutinizing procedure to ternary QWL coatings laid on a fused-silica substrate (assumed to be of infinite thickness), using $\mathrm{SiO}_{2}$ and $\mathrm{TiO}_{2}:: \mathrm{Ta}_{2} \mathrm{O}_{5}$ for the low-index $(L)$ and highindex $(H)$ materials, respectively. For illustrative purposes, we shall consider first two putative candidates for the third (high index) material $\left(H^{\prime}\right)$. These will be referred to as material A and material $\mathrm{B}$ and represent two rather extreme paradigms, 
similar to those discussed in Ref. [13]. Numerical experiments based on realistic materials, namely, $a \mathrm{Si}$ and $\mathrm{SiN}_{x}$, are presented in Sec. V.

Material A has the same mechanical losses as $\mathrm{TiO}_{2}:$ $\mathrm{Ta}_{2} \mathrm{O}_{5}$ and a fairly higher refractive index, but it has larger optical losses; material $\mathrm{B}$ has the same refractive index as $\mathrm{TiO}_{2}:: \mathrm{Ta}_{2} \mathrm{O}_{5}$ and fairly lower mechanical losses, but it has larger optical losses. The numerical values of the relevant properties of putative materials A and B are collected in Table I.

We consider different values of the extinction coefficient ranging from $10^{-6}$ to $10^{-4}$.

We use the following bounds in the transmittance and absorbance constraints (16):

$$
\tau_{\text {ref }}=6 \mathrm{ppm}, \quad \alpha_{\text {ref }}=1 \mathrm{ppm},
$$

and scale the loss angle of the various admissible solutions to that of a reference LIGO- or Virgo-like design, consisting of $N_{T}=36$ alternating titania-doped-tantala/silica layers, for which

$$
\phi_{C}=\phi_{C}^{(\mathrm{ref})}=18 \frac{\eta_{L} \lambda_{0}}{4 \operatorname{Re}\left(n_{L}\right)}\left(1+\gamma_{H}\right)
$$

\section{A. Ternary QWL coatings using material A}

The set of all admissible ternary QWL coatings compliant with the prescribed transmittance and absorbance constraints (21) and using material A for $H^{\prime}$ is collected in Table II for three possible values of the extinction coefficient $\left(\kappa_{H^{\prime}}=10^{-6}, 10^{-5}, 10^{-4}\right)$.

They are conveniently grouped into subsets featuring the same number of $[H \mid L],\left[H^{\prime} \mid L\right]$, and $\left[H^{\prime} \mid H\right]$ doublets, and hence the same coating loss angle, in order of increasing transmittance and/or absorbance. For all considered $\kappa_{H^{\prime}}$ values, the optimal design featuring the lowest coating loss angle consists of a stack of $N_{H^{\prime}}$ doublets $\left[H^{\prime} \mid L\right]$ grown on top of the substrate, topped by another stack of $N_{H}$ doublets $[H \mid L]$, where the optimal values of $N_{H^{\prime}}$ and $N_{H}$ depend on the extinction coefficient of the $H^{\prime}$ material.

There are no $\left[H^{\prime} \mid H\right]$ (or $\left[H \mid H^{\prime}\right]$ ) doublets in the optimal designs.

These findings confirm the heuristic assumption first made in Refs. [13,14] about the structure of ternary QWL coatings yielding minimal noise under prescribed transmittance and absorbance constraints.

\section{B. Ternary QWL coatings using material B}

In the case of ternary QWL coatings using material B, materials $H$ and $H^{\prime}$ are isorefractive; hence the total number of high-low-index doublets needed to satisfy the prescribed transmittance constraint remains fixed, irrespective of whether the high-index layers consist of the $H$ or $H^{\prime}$ material, and is the same as for the reference $\mathrm{TiO}_{2}:: \mathrm{Ta}_{2} \mathrm{O}_{5} / \mathrm{SiO}_{2}$ binary coating. Hence

$$
N_{H}+N_{H^{\prime}}=N_{L}=N_{L}^{(\mathrm{ref})} .
$$

Note also that in this case, $\left[H^{\prime} \mid H\right]$ (or $\left[H \mid H^{\prime}\right]$ ) doublets are forbidden, being optically homogeneous and half wavelength thick. (a)

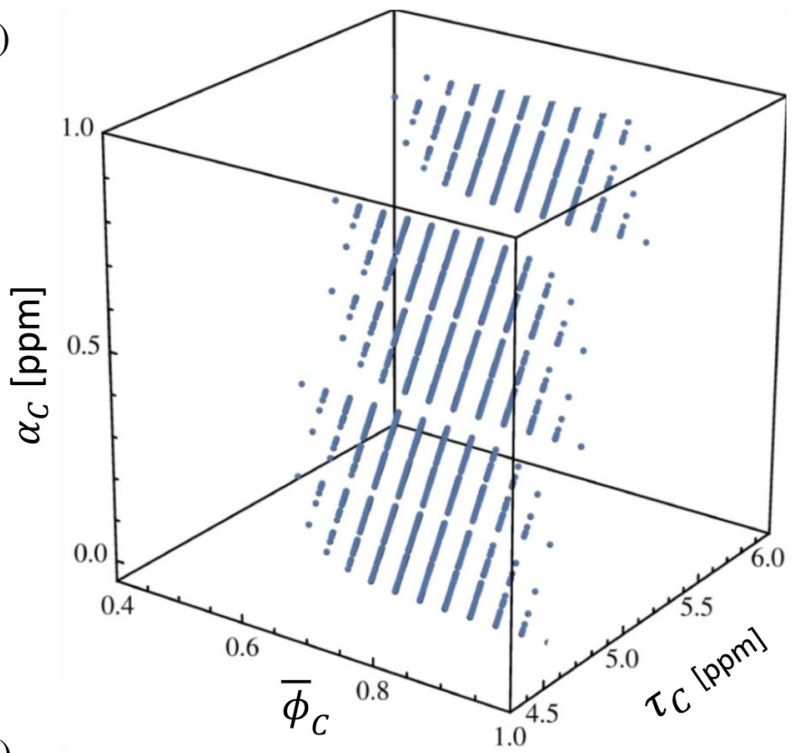

(b)

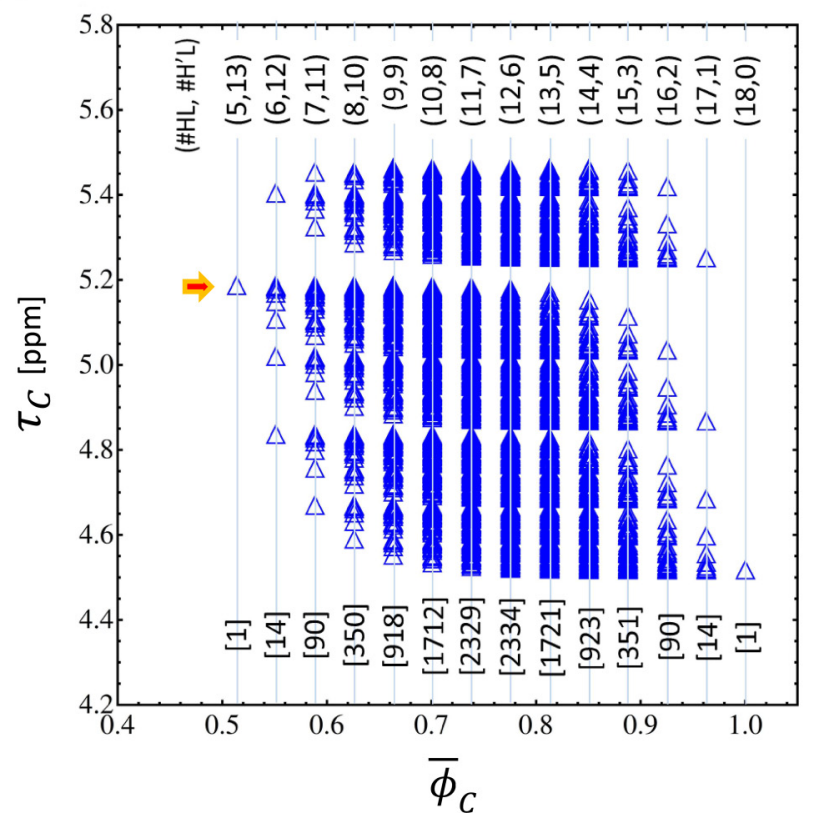

FIG. 2. The set of all admissible ternary QWL coatings subject to (16) using silica for $L$, titania-doped tantala for $H$, and material B for $H^{\prime}$, with $\kappa_{H^{\prime}}=10^{-5}$ and $\bar{\phi}_{C}=\phi_{C} / \phi_{C}^{(\text {ref })}$. See Sec. III for details.

The set of all admissible ternary QWL coatings compliant with the prescribed transmittance and absorbance constraints (21) can be conveniently visualized as in Fig. 2, which refers to the case $\kappa_{H^{\prime}}=10^{-5}$.

It is seen that all suboptimal, constraint-compliant admissible designs can be divided into distinct families, represented by the aligned markers in Figs. 2(a) and 2(b), each family featuring a number $\left(N_{H}\right)$ of $[H \mid L]$ doublets, denoted as \#HL in Fig. 2(b), and a fixed number $\left(N_{H^{\prime}}=N_{L}-N_{H}\right)$ of $\left[\left.H\right|^{\prime} L\right]$ doublets, denoted as $\# H^{\prime} L$ in Fig. 2(b); hence each family exhibits the same loss angle but different transmittances and absorbances, as seen from Fig. 2(a).

For each family, the number of distinct admissible designs, in square brackets in Fig. 2(b), is (slightly) less than the 
(a)

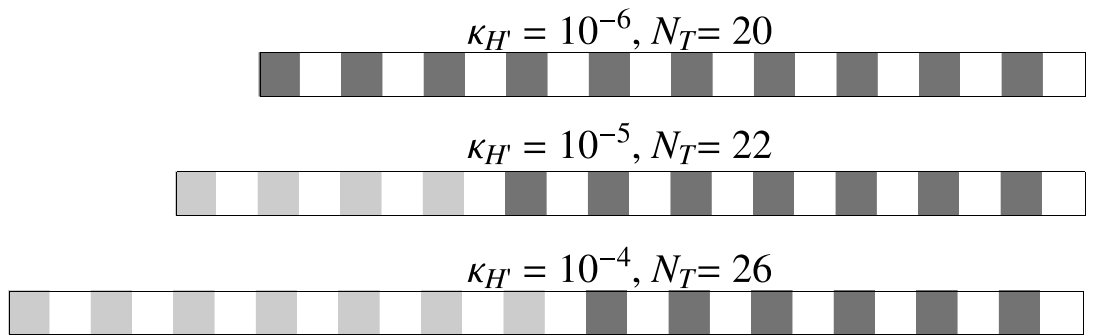

Optimal ternary QWL coatings using Material-A

\begin{tabular}{|c|c|c|c|}
\hline$\kappa_{H}$ & $\tau_{C}[\mathrm{ppm}]$ & $\alpha_{C}[\mathrm{ppm}]$ & $\phi_{C} / \phi_{C}^{(\mathrm{ref})}$ \\
\hline $10^{-6}$ & 2.246 & 0.911 & 0.377 \\
\hline $10^{-5}$ & 5.934 & 0.522 & 0.486 \\
\hline $10^{-4}$ & 3.074 & 0.564 & 0.615 \\
\hline
\end{tabular}

(b)

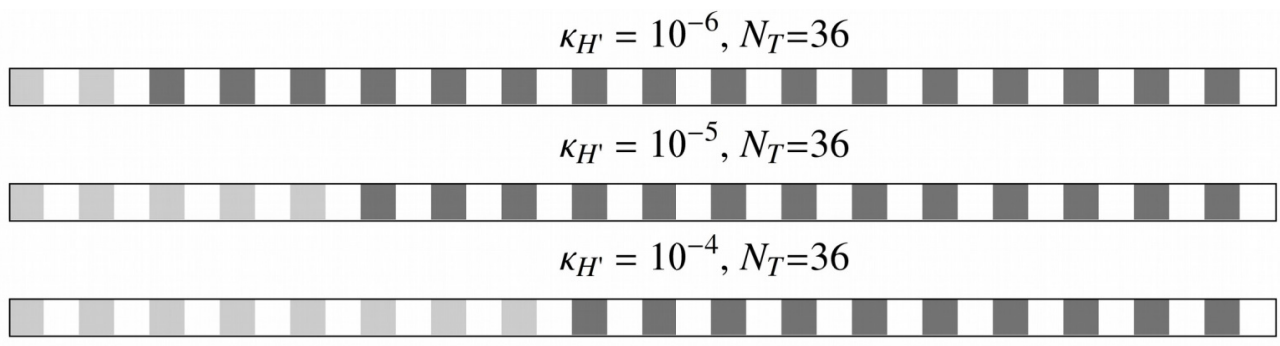

Optimal ternary QWL coatings using Material-B

\begin{tabular}{|c|c|c|c|}
\hline$\kappa_{H^{\prime}}$ & $\tau_{C}[\mathrm{ppm}]$ & $\alpha_{C}[\mathrm{ppm}]$ & $\phi_{C} / \phi_{C}^{(\mathrm{ref})}$ \\
\hline $10^{-6}$ & 5.128 & 0.661 & 0.401 \\
\hline $10^{-5}$ & 5.191 & 0.724 & 0.514 \\
\hline $10^{-4}$ & 5.248 & 0.781 & 0.626 \\
\hline
\end{tabular}

FIG. 3. The found optimal (minimum Brownian noise) QWL ternary coating designs subject to (16), using silica $(L)$, titania-doped tantala $(H)$, and (a) material A or (b) material B $\left(H^{\prime}\right)$, assuming different values for $\kappa_{H^{\prime}}$ (substrate on the right). White boxes, L; light gray boxes, $\mathrm{H}$, dark gray boxes, $\mathrm{H}^{\prime}$.

binomial coefficient

$$
\left(\begin{array}{c}
N_{H} \\
N_{L}-N_{H}
\end{array}\right)
$$

due to the (relatively few) designs that do not fulfill the transmittance and absorbance constraints.

Similar to the previous case, the optimal design featuring the lowest thermal noise under the prescribed transmittance and absorbance constraints consists of a stack of $\left[H^{\prime} \mid L\right]$ doublets grown on top of the substrate, topped by another stack of $[H \mid L]$ doublets, again confirming the ansatz in Refs. $[13,14]$.
The found optimal designs using materials $\mathrm{A}$ and $\mathrm{B}$ for $H^{\prime}$, with $\kappa_{H^{\prime}}=10^{-6}, 10^{-5}, 10^{-4}$, are displayed in Figs. 3(a) and 3(b), respectively.

\section{Robustness}

The optimal ternary QWL coatings are nicely robust against uncertainties in the value of the extinction coefficient $\kappa_{H^{\prime}}$, as well as against unavoidable inaccuracies in the layers' thicknesses, due to technological limitations of the deposition process [32]. 
Optimal ternary coating using Material-A
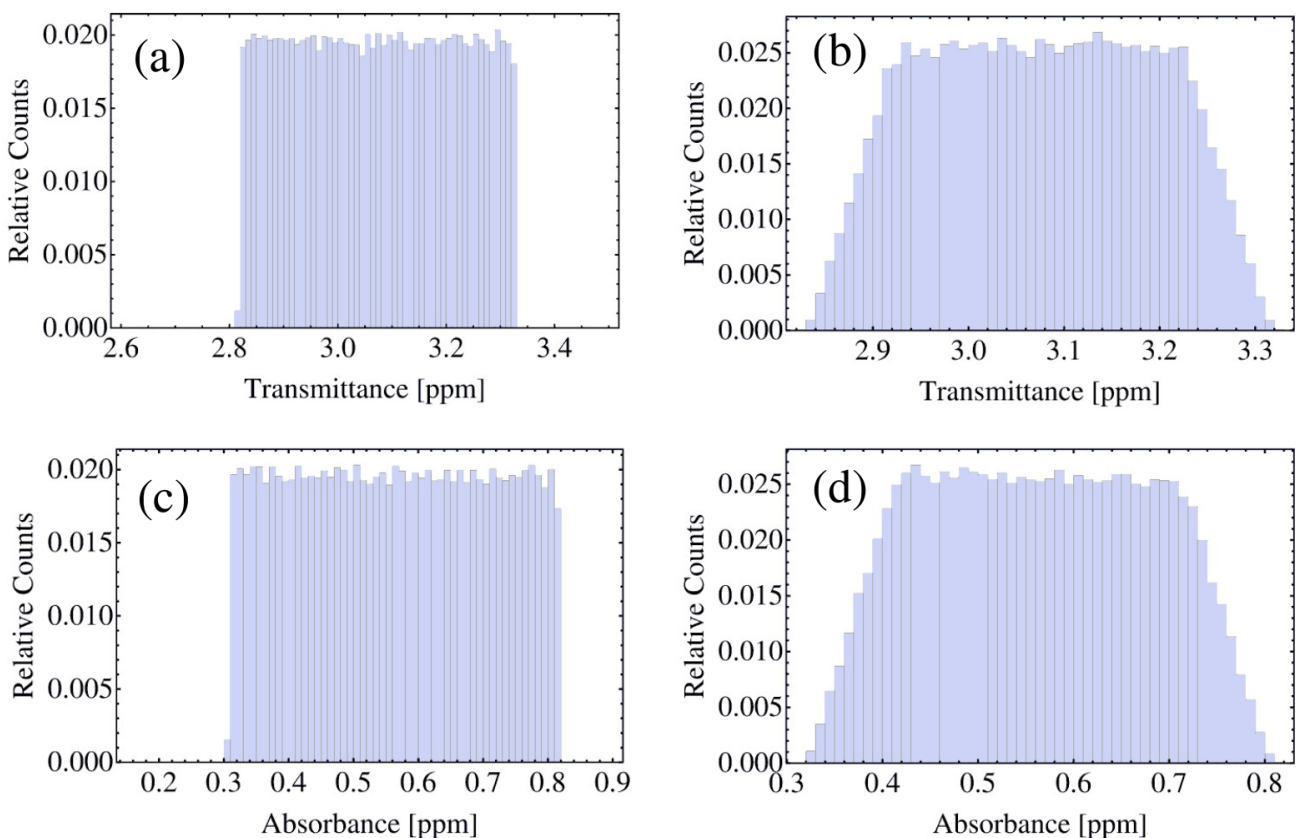

FIG. 4. Distributions of coating transmittance and absorbance in a sample of $10^{5}$ realizations of the optimal ternary QWL coating using material A, assuming $\kappa_{A}$ to be random uniform in $\left(0.5 \bar{\kappa}_{A}, 1.5 \bar{\kappa}_{A}\right)$, with $\bar{\kappa}_{A}=10^{-4}$. (a) and (c) refer to case (i), where the extinction coefficient is the same for all $H^{\prime}$ layers; (b) and (d) refer to case (ii), where the extinction coefficients of the $H^{\prime}$ layers are independent identically distributed random variables.

Optimal ternary coating using Material-B
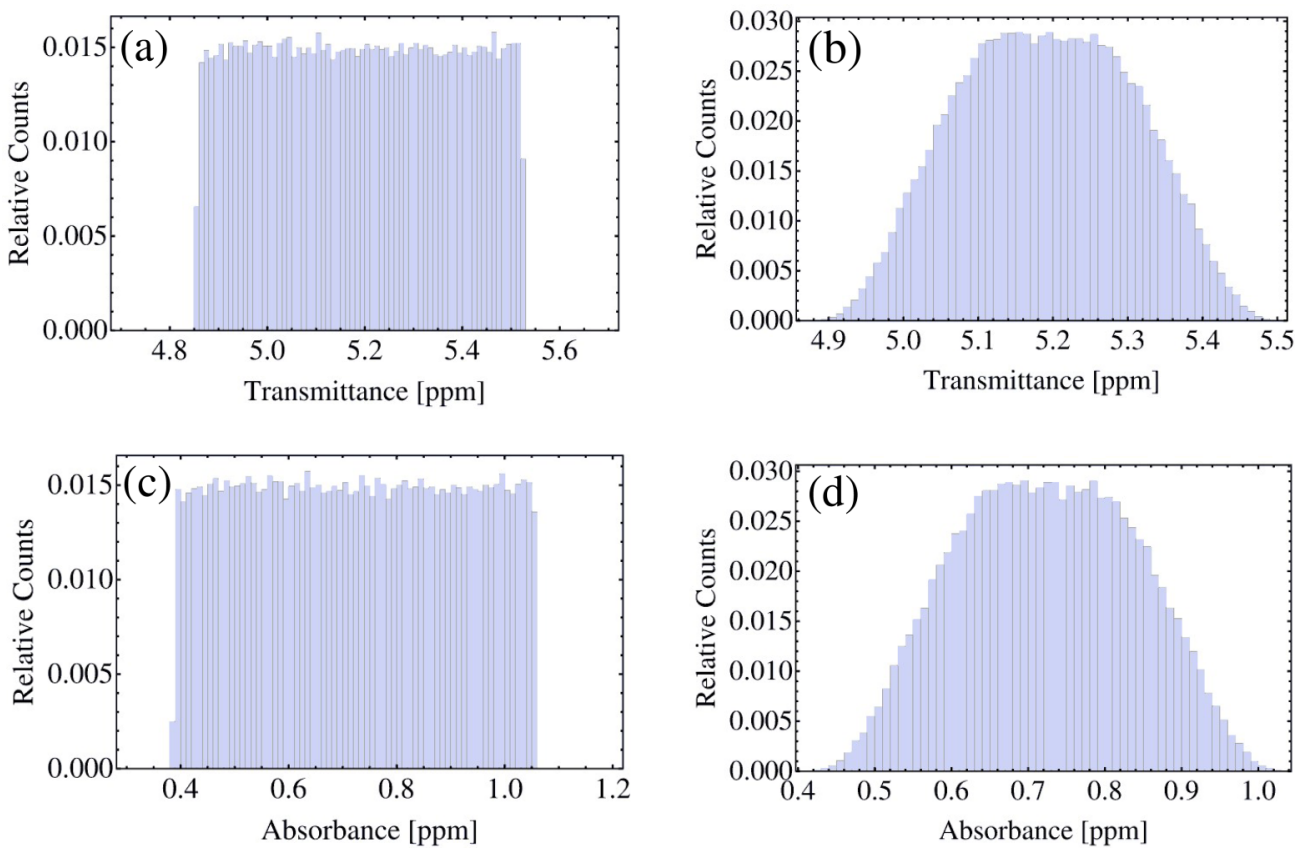

FIG. 5. Distributions of coating transmittance and absorbance in a sample of $10^{5}$ realizations of the optimal ternary QWL coating using material B, assuming $\kappa_{B}$ to be random uniform in $\left(0.5 \bar{\kappa}_{B}, 1.5 \bar{\kappa}_{B}\right)$, with $\bar{\kappa}_{B}=10^{-5}$. (a) and (c) refer to case (i), where the extinction coefficient is the same for all $H^{\prime}$ layers; (b) and (d) refer to case (ii), where the extinction coefficients of the $H^{\prime}$ layers are independent identically distributed random variables. 

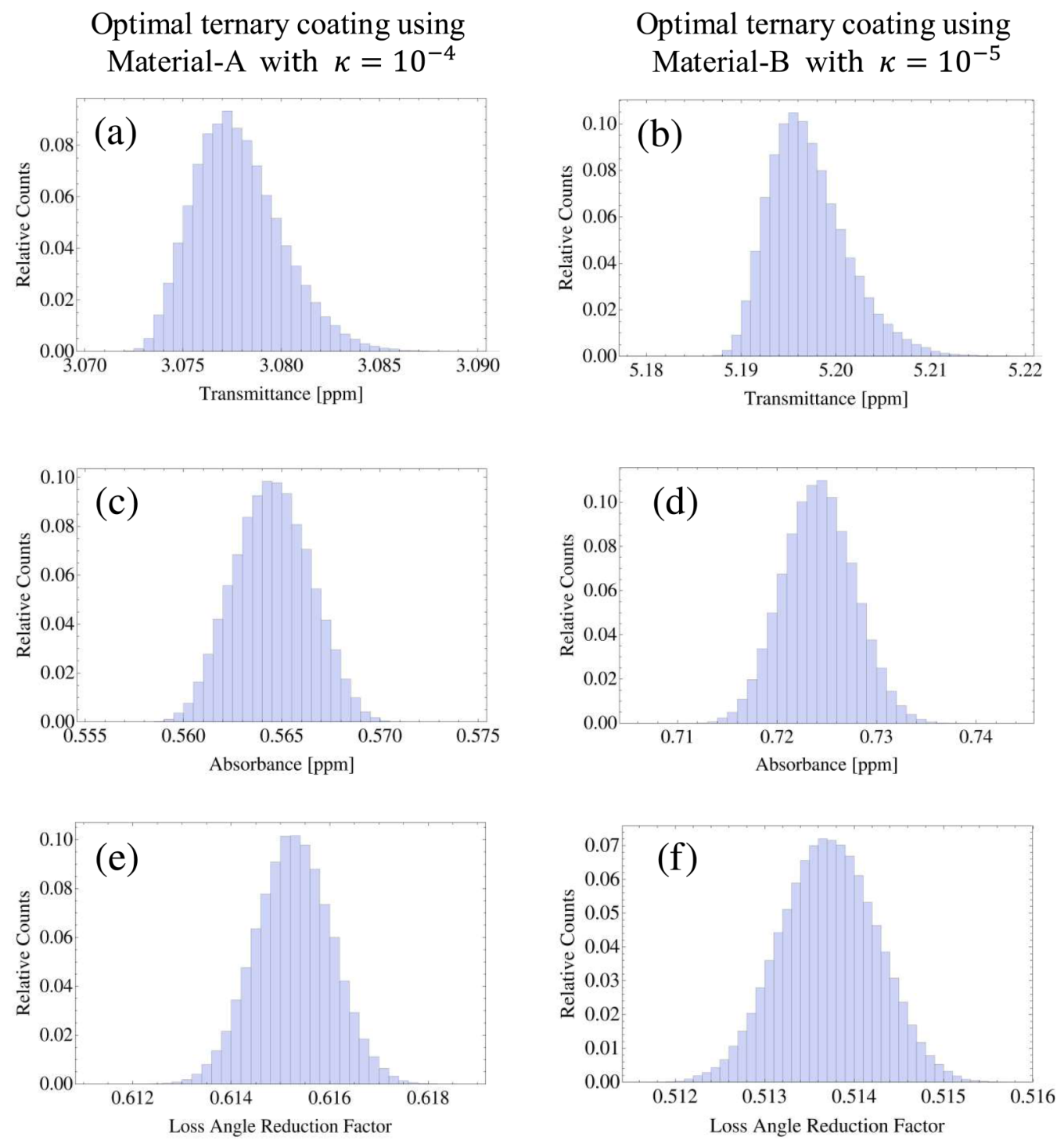

FIG. 6. Distributions of coating transmittance, absorbance, and loss angle (normalized to the value of the reference binary coating) in a sample of $10^{5}$ realizations, assuming the thicknesses of all layers to be independent random variables identically distributed uniformly around the nominal QWL thickness, in a symmetric interval of total width $2 \mathrm{~nm}$. (a), (c), and (e) Optimal ternary QWL coating using material A for $H^{\prime}$, with $\kappa_{A}=10^{-4}$. (b), (d), and (f) Optimal ternary QWL coating using material B for $H^{\prime}$, with $\kappa_{B}=10^{-5}$.

As an illustration, Fig. 4 shows the distributions of coating transmittances and absorbances in $10^{5}$ realizations of the optimal ternary QWL coating using material A.

Figures 4(a) and 4(c) refer to case (i), where the extinction coefficient $\kappa_{A}$ is the same for all $H^{\prime}$ layers and is random uniformly distributed in $\left(0.5 \bar{\kappa}_{A}, 1.5 \bar{\kappa}_{A}\right)$, with $\bar{\kappa}_{A}=10^{-4}$; Figs. 4(b) and 4(d) refer to case (ii), where the extinction coefficients of the $H^{\prime}$ layers are independent random variables, identically distributed as in case (i).

Similarly, Fig. 5 shows the distributions of coating transmittance and absorbance in a sample of $10^{5}$ realizations of the optimal ternary QWL coating using material $\mathrm{B}$, assuming $\kappa_{B}$ to be random uniformly distributed in $\left(0.5 \bar{\kappa}_{B}, 1.5 \bar{\kappa}_{B}\right)$, with $\bar{\kappa}_{B}=10^{-5}$.

Also in this case, Figs. 5(a) and 5(c) refer to the case where the random extinction coefficient is the same for all $H^{\prime}$ layers; Figs. 5(b) and 5(d) refer to the case where the extinction coefficients of the $H^{\prime}$ layers are independent and identically distributed as specified.

Not unexpectedly, uncertainties in the extinction coefficient stemming from fluctuations in the deposition process, rather than systematic uncertainty in the nominal value, have a lesser effect, due to possible fluctuation compensation, resulting in narrower distributions of the coating transmittance and absorbance.

Figure 6 shows the distributions of coating transmittance, absorbance, and loss angle (normalized to the value of the reference binary coating) in a sample of $10^{5}$ realizations of (i) the optimal ternary QWL coating using material A for $H^{\prime}$, with $\kappa_{A}=10^{-4}$ [Figs. 6(a), 6(c), and 6(e)], and (ii) the optimal ternary QWL coating using material B for $H^{\prime}$, with $\kappa_{B}=10^{-5}$ [Figs. 6(b), 6(d), and 6(f)], assuming the thicknesses of all layers to be independent random variables identically distributed uniformly around the nominal QWL thickness, in a symmetric interval of total width $2 \mathrm{~nm}$. 

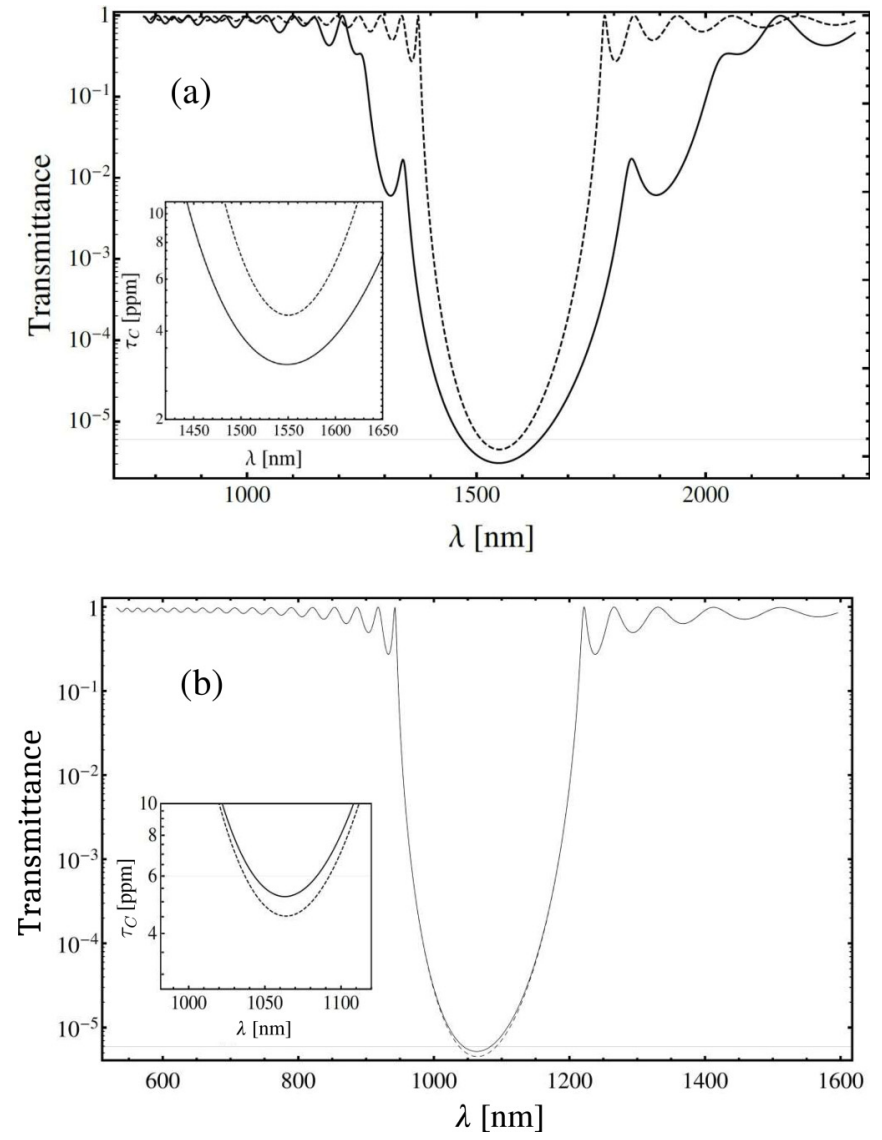

FIG. 7. Transmittance spectra of optimal designs using material A with $\kappa_{H^{\prime}}=10^{-4}$ and material B with $\kappa_{H^{\prime}}=10^{-5}$, respectively, in (a) and (b), for material $H^{\prime}$. Close-ups are shown in insets.

We may conclude that the optimal ternary QWL designs are fairly robust against uncertainties in the extinction coefficient of the $H^{\prime}$ material and deposition-related thickness errors.

\section{Transmittance spectra}

We computed the transmittance spectra of the above optimal ternary QWL coatings. These are shown in Figs. 7(a) and 7(b) for coatings using materials A and B, respectively. The spectra were computed neglecting chromatic dispersion, except in the neighborhood of the operating wavelength $\left(\lambda_{0}=\right.$ $1064 \mathrm{~nm})$, shown in the insets, where a linear approximation was used,

$$
n_{r}(\lambda)=n_{r}\left(\lambda_{0}\right)+\left.\frac{d n_{r}}{d \lambda}\right|_{\lambda_{0}}\left(\lambda-\lambda_{0}\right) .
$$

The pertinent values of $\left.\frac{d n_{r}}{d \lambda}\right|_{\lambda_{0}}$ were taken from Ref. [33] and are $-1.2 \times 10^{-5} \mathrm{~nm}^{-1}$ for silica, $-4.9 \times 10^{-5} \mathrm{~nm}^{-1}$ for titania-doped tantala, $-3.8 \times 10^{-5} \mathrm{~nm}^{-1}$ for material $\mathrm{A}$, and $-4.26 \times 10^{-4} \mathrm{~nm}^{-1}$ for material B.

Within the limits of this model, no ripple is observed in the high-reflectance band. The shape of the transmission spectrum for the optimal coating using material A departs more markedly from the reference spectrum compared with that of the optimal coating using material B. The observed asymmetry of the lobes stems from the fact that the coating is piecewise homogeneous, consisting of two cascaded homogeneous QWL stacks.

\section{E. Thickness optimization}

Thermal noise in binary coatings can be effectively reduced compared with the reference QWL-layer design by suitably reducing the total thickness of the mechanically noisier material(s), while increasing the total thickness of the other material(s) and the total number of doublets, so as to keep the coating transmittance unchanged [18].

Remarkably, the thickness-optimized binary coatings turn out to consist of almost identical stacked $[H \mid L]$ doublets whose thickness is one-half of the working wavelength (Bragg condition), the exception being represented by a few layers near the coating top and bottom [34-37].

Implementing thickness optimization for ternary coatings is computationally demanding. In a full-blind exhaustive approach, each and any $L$ layer should be allowed to take any thickness value in the range $(\lambda / 4, \lambda / 2)$, and each and any $H$ and $H^{\prime}$ layer should be allowed to take any thickness in $(0, \lambda / 4), \lambda$ being the local wavelength. Even after suitable discretization of the above search intervals, the computational burden of an exhaustive search would become prohibitive for any meaningful value of $N_{T}$.

A reasonable heuristic approach to thickness optimization of ternary QWL coatings may thus consist in optimizing the two binary QWL stacks that form the top and bottom parts of the optimal QWL ternary coatings, assuming each of them to consist of identical non-QWL Bragg doublets [38].

Here, to illustrate the possible margins of further loss angle reduction obtainable from thickness optimization, we content ourselves with computing the (normalized) coating loss angle, absorbance, and power transmittance of the coatings obtained

TABLE III. Thickness-tweaked optimal ternary QWL designs.

\begin{tabular}{lcrr}
\hline \hline & Configuration & $\tau_{c}(\mathrm{ppm})$ & $\alpha_{c}(\mathrm{ppm})$ \\
\hline Material A & QWL, $[(H \mid L)]^{6}\left[\left(H^{\prime} \mid L\right)\right]^{6}$ & 0.564 \\
$\left(\right.$ with $\left.k_{H^{\prime}}=10^{-4}\right)$ & $d_{L}=\lambda / 3 ; d_{H^{\prime}}=\lambda / 6$ & 3.074 & 0.822 \\
& {$[(H \mid L)]^{9}\left[\left(H^{\prime} \mid L\right)\right]^{9}$} & 2.963 & 0.615 \\
\hline Material B & $\mathrm{QWL},[(H \mid L)]^{5}\left[\left(H^{\prime} \mid L\right)\right]^{13}$ & & 0.549 \\
(with $\left.k_{H^{\prime}}=10^{-5}\right)$ & $d_{L}=\lambda / 3 ; d_{H^{\prime}}=\lambda / 6$ & 5.191 & 0.724 \\
& {$[(H \mid L)]^{5}\left[\left(H^{\prime} \mid L\right)\right]^{16}$} & 4.801 & 0.982 \\
\hline
\end{tabular}


TABLE IV. Numerical values of relevant parameters for realistic candidate ternary coating materials and substrates, at selected temperatures and wavelengths. All symbols have the usual meaning. Substrate: silica at $1064 \mathrm{~nm}(n=1.44 ; Y=72$ GPa $)$; crystalline silicon $^{\mathrm{f}}$ at $1550 \mathrm{~nm}(n=3.58 ; Y=160 \mathrm{GPa})$. NIR $=$ near infrared.

\begin{tabular}{|c|c|c|c|c|}
\hline & $n$ & $\kappa$ & $\phi$ & $Y(\mathrm{Gpa})$ \\
\hline$a \mathrm{Si}^{\mathrm{a}, \mathrm{b}}$ & 3.5 at $1550 \mathrm{~nm}$ & $1.22 \times 10^{-5}$ at $1550 \mathrm{~nm}$ & $\begin{array}{c}10^{-4} \text { at } 290 \mathrm{~K} \\
8 \times 10^{-5} \text { at } 120 \mathrm{~K} \\
2 \times 10^{-5} \text { at } 20 \mathrm{~K}\end{array}$ & 147 \\
\hline $\mathrm{SiN} x^{c, d}$ & 2.28 at $1064 \mathrm{~nm}$ & $1.51 \times 10^{-5}$ at $1550 \mathrm{~nm}$ & $\begin{array}{c}8 \times 10^{-5} \text { at } 290 \mathrm{~K} \\
2 \times 10^{-5} \text { at } 120 \mathrm{~K} \\
1 \times 10^{-5} \text { at } 20 \mathrm{~K}\end{array}$ & 103.7 \\
\hline $\mathrm{SiO}_{2}{ }^{\mathrm{e}}$ & 1.44 (NIR) & $8 \times 10^{-8}$ & $\begin{array}{c}5 \times 10^{-5} \text { at } 290 \mathrm{~K} \\
1.7 \times 10^{-4} \text { at } 120 \mathrm{~K} \\
7.8 \times 10^{-4} \text { at } 20 \mathrm{~K}\end{array}$ & 72 \\
\hline $\mathrm{TiO}_{2}:: \mathrm{Ta}_{2} \mathrm{O}_{5}^{\mathrm{e}}$ & 2.05 (NIR) & $8 \times 10^{-8}$ & $\begin{array}{c}3.66 \times 10^{-4} \text { at } 290 \mathrm{~K} \\
\text { Interpolated at } 120 \mathrm{~K} \\
8.6 \times 10^{-4} \text { at } 20 \mathrm{~K}\end{array}$ & 140 \\
\hline
\end{tabular}

${ }^{\mathrm{a}}$ Ref. [39]; ${ }^{\mathrm{b}}$ Ref. [40]; ${ }^{\mathrm{c}}$ Ref. [41]; ${ }^{\mathrm{d}}$ Ref. [10]; ${ }^{\mathrm{e}}$ Ref. [42]; ${ }^{\mathrm{f}}$ Ref. [43].

after modifying the optimal ternary QWL coatings found in the previous sections and listed in Fig. 3 by letting

$$
\frac{n_{H} d_{H}}{\lambda_{0}}=\frac{n_{H}^{\prime} d_{H}^{\prime}}{\lambda_{0}}=\frac{1}{6} \quad \text { and } \quad \frac{n_{L} d_{L}}{\lambda_{0}}=\frac{1}{3}
$$

so as to preserve the Bragg character of the doublets and adding a few $[H \mid L]$ and/or $\left[H^{\prime} L\right]$ layers as needed to maintain compliance with the transmittance and absorbance constraints.

The transmittance, absorbance, and coating loss angle (normalized to the reference value) of the resulting thicknesstweaked coatings are collected in Table III.

\section{REALISTIC MATERIALS}

The structure and properties of the optimal coatings are the same if we consider realistic candidates for the third material, namely, $a \mathrm{Si}$ and $\mathrm{SiN}_{x}$.

In this section we present results based on the previous analysis or simulation tools for optimal QWL ternary coatings operating at 290, 120, and $20 \mathrm{~K}$, based (i) on silica, titania-doped tantala, and $a \mathrm{Si}$ at $1550 \mathrm{~nm}$ and (ii) on silica, titania-doped tantala, and $\mathrm{SiN}_{x}$ at $1064 \mathrm{~nm}$, using current available measurements or estimates of the actual (or fiducially achievable) material parameters, collected in Table IV.

The coating Brownian noise power spectral density (PSD) reduction factor with respect to the reference advanced LIGO (aLiGO) and advanced Virgo (adVirgo) coatings currently in operation (at ambient temperature) is shown in Figs. 8(a) and 8 (b), respectively, for different values of the extinction coefficient of the third material, in the range from $10^{-5}$ to $10^{-4}$. In calculating the Brownian noise PSD reduction factor we obviously include the temperature-dependent factor in Eq. (13). We note in passing that reducing the PSD by a factor $\rho$ corresponds to reducing the so-called amplitude spectral density (or rms noise level) by a factor $\rho^{1 / 2}$ and to boosting the visibility distance by a factor $\rho^{-1 / 2}$.

The integer pairs $(M, N)$ in parentheses in Fig. 8 represent the numbers of $[H \mid L]$ and $\left[H^{\prime} \mid L\right]$ doublets in the top and bottom stacks of the found optimal ternary QWL design featuring the minimal thermal noise under the prescribed constraints $\tau_{C} \leqslant 6 \mathrm{ppm}$ and $\alpha_{C} \leqslant 1 \mathrm{ppm}$.

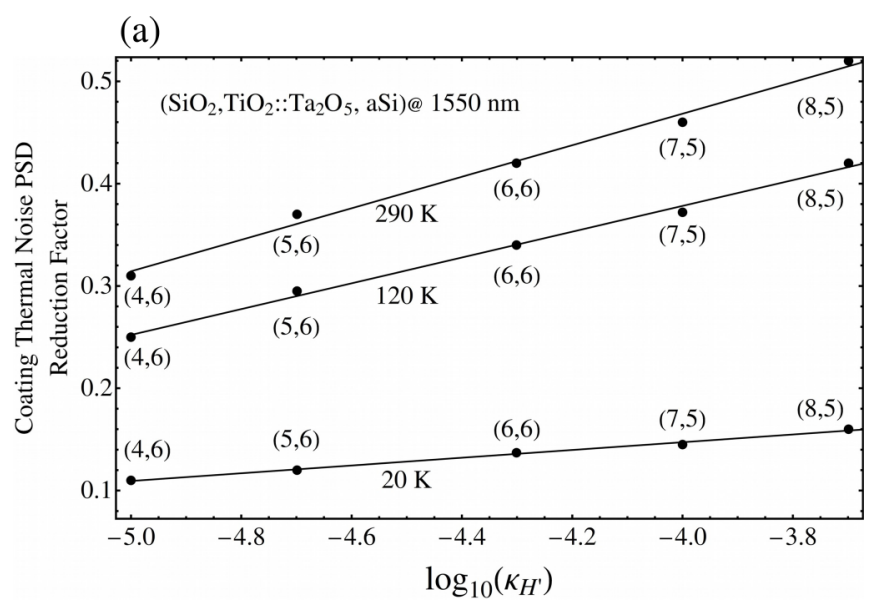

(b)

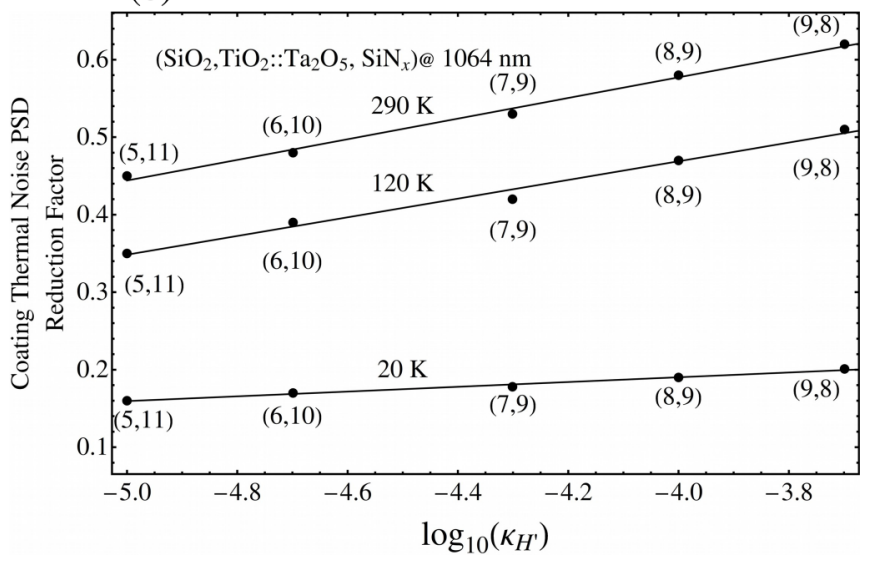

FIG. 8. Achievable thermal (Brownian) noise reduction factors for optimal QWL ternary coatings vs extinction coefficient of third $\left(H^{\prime}\right)$ material. The coatings in (a) use $a \mathrm{Si}$ (at $1550 \mathrm{~nm}$ ) and those in (b) use $\mathrm{SiN}_{x}$ (at $1064 \mathrm{~nm}$ ) as the third material, together with $\mathrm{SiO}_{2}$ and $\mathrm{Ta}_{2} \mathrm{O}_{5}$. Three different operating temperatures $(290,120$, and $20 \mathrm{~K}$ ) are considered. 
Remarkably, as seen from the figure, the thermal noise reduction factor is found to be almost linear in $\log _{10}\left(\kappa_{H^{\prime}}\right)$.

The achievable coating thermal noise PSD reduction factor is nicely large, especially at cryogenic temperatures, where it gets close to the Einstein Telescope [44] and Cosmic Explorer [45] requirements and is comparable to the expected performance of crystalline coatings [46], while possibly posing less demanding technological challenges.

\section{CONCLUSIONS}

In this paper we addressed the problem of designing a ternary optical coating consisting of QWL layers to achieve the minimum thermal (Brownian) noise under prescribed optical transmittance and absorbance constraints.

We first considered ternary coatings where two materials are those presently in use in the advanced LIGO and Virgo detectors, namely, $\mathrm{SiO}_{2}$ and $\mathrm{TiO}_{2}:: \mathrm{Ta}_{2} \mathrm{O}_{5}$, featuring the best trade-off between optical contrast, optical losses, and thermal noise so far, and the third material is one of two putative materials featuring, respectively, the same mechanical losses as $\mathrm{TiO}_{2}:: \mathrm{Ta}_{2} \mathrm{O}_{5}$ and a higher refractive index, but larger optical losses (material A), or the same refractive index as $\mathrm{TiO}_{2}:: \mathrm{Ta}_{2} \mathrm{O}_{5}$ and fairly lower mechanical losses, but larger optical losses (material B), allowing their extinction coefficient to range from $10^{-6}$ to $10^{-4}$ in both cases.

We performed an exhaustive search over all possible (and admissible, in the sense discussed in Sec. IV) configurations consisting of QWL layers, using backtracking for numerical efficiency and seeking the optimal designs yielding minimum thermal (Brownian) noise under prescribed upper bounds for transmittance and absorbance. lows.

The main results of this study can be summarized as fol-

All found optimal designs consist of a stack of $\left[H^{\prime} \mid L\right]$ doublets grown on top of the substrate, topped by another stack of $[H \mid L]$ doublets, confirming the ansatz in Refs. $[13,14]$.
They are nicely robust against deposition inaccuracies in the individual layer thicknesses and systematic uncertainties and/or fluctuations from layer to layer of the extinction coefficient. Their transmittance spectra satisfy the design constraints in the useful band.

We have further shown that a further improvement in performance can be achieved by using thinner layers of the noisier materials in each Bragg doublet. Exhaustive blind thickness optimization of ternary coatings appears to be computationally unaffordable, though, and further study of the problem following a heuristic approach is needed [47].

Next we applied the same optimization strategy to realistic candidates for the third material, namely, $a \mathrm{Si}$ and $\mathrm{SiN}_{x}$, operating at three different temperatures $(290,120$, and $20 \mathrm{~K})$. In order to take into account the present uncertainties (and possible margins of improvement [39]) for the optical losses of these materials, we considered different values of their extinction coefficient in the range from $10^{-6}$ to $10^{-4}$.

All optimal designs outperform significantly the reference binary solution consisting of alternating QWL layers made of silica and titania-doped tantala, in terms of Brownian thermal noise.

In particular, according to our simulations, QWL ternary coatings using either $a \mathrm{Si}$ or $\mathrm{SiN}_{x}$ in addition to silica and titania-doped tantala may achieve an almost tenfold reduction in the coating thermal noise (power spectrum) level compared with second-generation detector coatings operating at ambient temperature.

\section{ACKNOWLEDGMENTS}

This work has been supported in part by Istituto Nazionale di Fisica Nucleare (INFN) through the projects Virgo and ETItaly and by the European Gravitational Observatory (EGO). The authors gratefully acknowledge useful discussions with, and suggestions from, members of the Virgo Coating R\&D Group (VCR\&D) and the LIGO Optics Working Group (OWG).
[1] D. V. Martynov, E. D. Hall, B. P. Abbott, R. Abbott, T. D. Abbott, C. Adams, R. X. Adhikari, R. A. Anderson, S. B. Anderson, K. Arai, M. A. Arain, S. M. Aston, L. Austin, S. W. Ballmer, M. Barbet, D. Barker, B. Barr, L. Barsotti, J. Bartlett, M. A. Barton et al., Sensitivity of the Advanced LIGO detectors at the beginning of gravitational wave astronomy, Phys. Rev. D 93, 112004 (2016).

[2] G. M. Harry, M. R. Abernathy, A. E. Becerra-Toledo, H. Armandula, E. Black, K. Dooley, M. Eichenfield, C. Nwabugwu, A. Villar, D. R. M. Crooks, G. Cagnoli, J. Hough, C. R. How, I. MacLaren, P. Murray, S. Reid, S. Rowan, P. H. Sneddon, M. M. Fejer, R. Route et al., Titania-doped tantala/silica coatings for gravitational wave detectors, Classical Quantum Gravity 24, 405 (2007).

[3] J. Aasi, B. P. Abbott, R. Abbott, T. Abbott, M. R. Abernathy, K. Ackley, C. Adams, T. Adams, P. Addesso, R. X. Adhikari, V. Adya, C. Affeldt, N. Aggarwal, O. D. Aguiar, A. Ain, P. Ajith, A. Alemic, B. Allen, D. Amariutei, S. B. Anderson et al. (The LIGO Scientific Collaboration), Advanced LIGO, Classical Quantum Gravity 32, 074001 (2015).

[4] F. Acernese, M. Agathos, K. Agatsuma, D. Aisa, N. Allemandou, A. Allocca, J. Amarni, P. Astone, G. Balestri, G. Ballardin et al., Advanced Virgo: a second-generation interferometric gravitational wave detector, Classical Quantum Gravity 32, 024001 (2015).

[5] G. Vajente, Review of Amorphous Coatings, LIGO Document G1900400, 2019.

[6] J. Steinlechner, I. W. Martin, A. S. Bell, J. Hough, M. Fletcher, P. G. Murray, R. Robie, S. Rowan, and R. Schnabel, Silicon-Based Optical Mirror Coatings for Ultrahigh Precision Metrology and Sensing, Phys. Rev. Lett. 120, 263602 (2018).

[7] L. Terkowski, I. W. Martin, D. Axmann, M. Behrendsen, F. Pein, A. Bell, R. Schnabel, R. Bassiri, M. M. Fejer, J. Hough, A. Markosyan, S. Rowan, and J. Steinlechner, Influence of Deposition Parameters on the Optical Absorption of Amorphous Silicon Thin Films, Phys. Rev. Res. 2, 033308 (2020). 
[8] R. Schnabel, M. Britzger, F. Brückner, O. Burmeister, K. Danzmann, J. Duck, T. Eberle1, D. Friedrich, H. Luck, M. Mehmet, R. Nawrodt, S. Steinlechner, and B. Willke, Building blocks for future detectors: Silicon test masses and $1550 \mathrm{~nm}$ laser light, J. Phys.: Conf. Ser. 228, 012029 (2010).

[9] The use of $2-\mu \mathrm{m}$ wavelength lasers also been suggested [48], subject to substantial progress in the candidate sources [Tm:YAG or Ho:YAG lasers] in terms of power-noise trade-off.

[10] H. W. Pan, L. C. Kuo, L. A. Chang, S. Chao, I. W. Martin, J. Steinlechner, and M. Fletcher, Silicon nitride and silica quarterwave stacks for low-thermal-noise mirror coatings, Phys. Rev. D 98, 102001 (2018).

[11] J. Steinlechner, C. Kruger, I. W. Martin, A. Bell, J. Hough, H. Kaufer, S. Rowan, R. Schnabel, and S. Steinlechner, Optical absorption of silicon nitride membranes at $1064 \mathrm{~nm}$ and at 1550 nm, Phys. Rev. D 96, 022007 (2017).

[12] M. Granata, A. Amato, G. Cagnoli, M. Coulon, J. Degallaix, D. Forest, L. Mereni, C. Michel, L. Pinard, B. Sassolas, and J. Teillon, Progress in the measurement and reduction of thermal noise in optical coatings for gravitational-wave detectors, Appl. Opt. 59, A229 (2020).

[13] W. Yam, S. Gras, and M. Evans, Multimaterial coatings with reduced thermal noise, Phys. Rev. D 91, 042002 (2015).

[14] J. Steinlechner, I. W. Martin, J. Hough, C. Kruger, S. Rowan, and R. Schnabel, Thermal noise reduction and absorption optimization via multimaterial coatings, Phys. Rev. D 91, 042001 (2015).

[15] S. C. Tait, J. Steinlechner, M. M. Kinley-Hanlon, P. G. Murray, J. Hough, G. McGhee, F. Pein, S. Rowan, R. Schnabel, C. Smith, L. Terkowski, and I. W. Martin, Demonstration of the Multimaterial Coating Concept to Reduce Thermal Noise in Gravitational-Wave Detectors, Phys. Rev. Lett. 125, 011102 (2020).

[16] J. I. Larruquert, M. Vidal Dasilva, S. Garca-Cortés, M. Fernández-Perea, J. A. Méndez, and J. A. Aznárez, Constructing multilayers with absorbing materials, Chin. Opt. Lett. Suppl. 8, 159 (2010).

[17] S. J. Orfanidis, Electromagnetic Waves and Antennas, 2016, https://www.ece.rutgers.edu/ $\sim$ orfanidi/ewa/.

[18] I. M. Pinto, M. Principe, and R. DeSalvo, Reflectivity and thickness optimization, in Optical Coatings and Thermal Noise in Precision Measurements, edited by G. Harry, T. R. Bodiya, and R. DeSalvo (Cambridge University Press, Cambridge, 2012), Chap. 12, pp. 173-195.

[19] G. M. Harry, H. Armandula, E. Black, D. R. M. Crooks, G. Cagnoli, J. Hough, P. Murray, S. Reid, S. Rowan, P. Sneddon, M. M. Fejer, R. Route, and S. D. Penn, Thermal noise from optical coatings in gravitational wave detectors, Appl. Opt. 45, 1569 (2006).

[20] T. Hong, H. Yang, E. K. Gustafson, R. X. Adhikari, and Y. Chen, Brownian thermal noise in multilayer coated mirrors, Phys. Rev. D 87, 082001 (2013).

[21] Other options that also appear promising, though on different timescales, including wide beams, crystalline materials, nanolayered composites, coatingless mirrors, and diffractive optics [49] fall beyond the scope of this paper.

[22] I. Martin, H. Armandula, C. Comtet, M. M. Fejer, A. Gretarsson, G. Harry, J. Hough, J.-M. M. Mackowski, I. MacLaren, C. Michel, J.-L. Montorio, N. Morgado, R. Nawrodt, S. Penn, S. Reid, A. Remillieux, R. Route, S. Rowan, C.
Schwarz, P. Seidel et al., Measurements of a low-temperature mechanical dissipation peak in a single layer of $\mathrm{Ta}_{2} \mathrm{O}_{5}$ doped with $\mathrm{TiO}_{2}$, Classical Quantum Gravity 25, 055005 (2008).

[23] I. Martin, R. Nawrodt, K. Craig, C. Schwarz, R. Bassiri, G. Harry, J. Hough, S. Penn, S. Reid, R. Robie, and S. Rowan, Low temperature mechanical dissipation of an ion-beam sputtered silica film, Classical Quantum Gravity 31, 035019 (2014).

[24] http://www.et-gw.eu/index.php.

[25] https://cosmicexplorer.org/.

[26] T. Akutsu and the KAGRA Collaboration, KAGRA: 2.5 generation interferometric gravitational wave detector, Nat. Astron. 3, 35 (2019).

[27] Note that expansion of the hyperbolic functions to first order in $\kappa^{(m)}$ (assumed to be $\ll 1$ for all materials involved) may lead to inaccuracies in the computed absorbances that could be nonnegligible comparable with the enforced absorbance bounds.

[28] Two consecutive QWL layers with the same refractive index would make a half-wavelength optically homogeneous layer, whose transmission matrix (2) is the identity matrix. Accordingly, such a doublet would not affect coating transmittance, while increasing coating thermal noise.

[29] This follows immediately by noting that the first layer can be chosen in three possible ways, while each of the $N_{T}-1$ subsequent ones can be chosen only in two possible ways, in view of the condition $n^{(m)} \neq n^{(m-1)}$.

[30] K. Ghedira and B. Dubuisson, Constraint Satisfaction Problems (John Wiley, New York, 2013).

[31] D. E. Knuth, The Art of Computer Programming (AddisonWesley, Reading, MA, 1968), Vol. 4 (5B).

[32] The assumed deposition errors are deemed as realistic; the assumed fluctuations in the extinction coefficient are mere bona fide values, due to the lack of available results in the literature.

[33] https://refractiveindex.info.

[34] J. Agresti, G. Castaldi, R. DeSalvo, V. Galdi, V. Pierro, and I. M. Pinto, Optimized multilayer dielectric mirror coatings for gravitational wave interferometers, Proc. SPIE 6286, 628608 (2006).

[35] A. E. Villar, E. D. Black, R. DeSalvo, K. G. Libbrecht, C. Michel, N. Morgado, L. Pinard, I. M. Pinto, V. Pierro, V. Galdi, M. Principe, and I. Taurasi, Measurement of thermal noise in multilayer coatings with optimized layer thickness, Phys. Rev. D 81, 122001 (2010).

[36] N. M. Kondratiev, A. G. Gurkovsky, and M. L. Gorodetsky, Thermal noise and coating optimization in multilayer dielectric mirrors, Phys. Rev. D 84, 022001 (2011).

[37] V. Pierro, V. Fiumara, F. Chiadini, F. Bobba, G. Carapella, C. Di Giorgio, O. Durante, R. Fittipaldi, E. Mejuto Villa, J. Neilson, M. Principe, and I. M. Pinto, On the performance limits of coatings for gravitational wave detectors made of alternating layers of two materials, Opt. Mater. 96, 109269 (2019).

[38] I. M. Pinto, Stacked-Triplet Ternary HR Coatings: Another Multimaterial Design Option, LIGO Document G2000218, 2020.

[39] R. Birney, J. Steinlechner, Z. Tornasi, S. MacFoy, D. Vine, A. S. Bell, D. Gibson, J. Hough, S. Rowan, P. Sortais, S. Sproules, S. Tait, I. W. Martin, and S. Reid, Amorphous Silicon with Extremely Low Absorption: Beating Thermal Noise in Gravitational Astronomy, Phys. Rev. Lett. 121, 191101 (2018). 
[40] P. G. Murray, I. W. Martin, K. Craig, J. Hough, R. Robie, S. Rowan, M. R. Abernathy, T. Pershing, and S. Penn, Ion-beam sputtered amorphous silicon films for cryogenic precision measurement systems, Phys. Rev. D 92, 062001 (2015).

[41] X. Liu, T. H. Metcalf, Q. Wang, and D. M. Photiadis, Elastic properties of several silicon nitride films, Mater. Res. Soc. Symp. Proc. 989, 2201 (2006).

[42] J. Steinlechner, I. W. Martin, R. Bassiri, A. Bell, M. M. Fejer, J. Hough, A. Markosyan, R. K. Route, S. Rowan, and Z. Tornasi, Optical absorption of ion-beam sputtered amorphous silicon coatings, Phys. Rev. D 93, 062005 (2016).

[43] M. A. Hopcroft, W. D. Nix, and T. W. Kenny, What is the Young's modulus of silicon? J. Microelectromech. Syst. 19, 229 (2010).

[44] The ET Steering Editorial Team, Einstein Telescope Design Report Update 2020, Sect. 6.3, Report No. ET-0007A-20, 2020.

[45] E. D. Hall, K. Kuns, J. R. Smith, Y. Bai, C. Wipf, S. Biscans, R. X. Adhikari, K. Arai, S. Ballmer, L. Barsotti, Y. Chen, M. Evans, P. Fritschel, J. Harms, B. Kamai, J. G. Rollins, D. Shoemaker, B. Slagmolen, R. Weiss, and H. Yamamoto,
Gravitational-wave physics with Cosmic Explorer: limits to low-frequency sensitivity, Sect. V. D, arXiv:2012.03608.

[46] G. Cole, W. Zhang, M. J. Martin, J. Ye, and M. Aspelmeyer, Tenfold reduction of Brownian noise in high-reflectivity optical coatings, Nat. Photonics 7, 644 (2013).

[47] V. Pierro, I. M. Pinto, Ternary Coatings Optimization, LIGO Document G2100429, 2021.

[48] R. X. Adhikari, K. Arai, A. F. Brooks, C. Wipf, O. Aguiar, P. Altin, B. Barr, L. Barsotti, R. Bassiri, A. Bell et al., A cryogenic silicon interferometer for gravitational-wave detection, Classical Quantum Gravity 37, 165003 (2020).

[49] M. Principe, Reflective Coating optimization for interferometric detectors of gravitational waves, Opt. Express 23, 10938 (2015).

Correction: A proof change request was misinterpreted by the production team and rendered errors in the affiliations for the first, sixth, eleventh, and twelfth authors. These affiliations have been fixed, and an additional affiliation has been inserted for the twelfth author. 